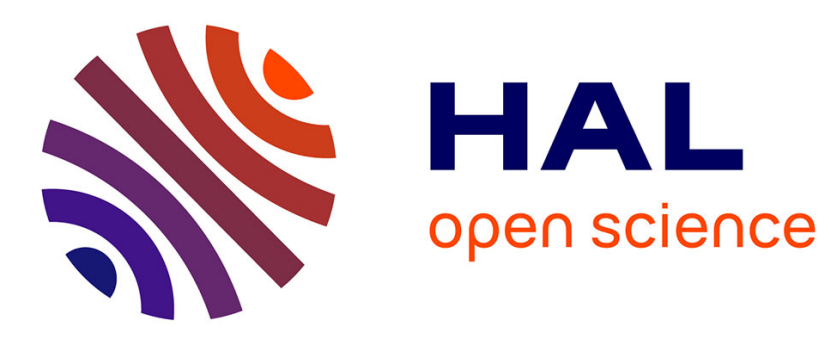

\title{
Études de sauts de flux limités dans les supraconducteurs de seconde espèce
}

\author{
L. Boyer, G. Fournet, A. Mailfert, J.L. Noel
}

\section{To cite this version:}

L. Boyer, G. Fournet, A. Mailfert, J.L. Noel. Études de sauts de flux limités dans les supraconducteurs de seconde espèce. Revue de Physique Appliquée, 1971, 6 (4), pp.501-504. 10.1051/rphysap:0197100604050100 . jpa-00243577

\section{HAL Id: jpa-00243577 https://hal.science/jpa-00243577}

Submitted on 1 Jan 1971

HAL is a multi-disciplinary open access archive for the deposit and dissemination of scientific research documents, whether they are published or not. The documents may come from teaching and research institutions in France or abroad, or from public or private research centers.
L'archive ouverte pluridisciplinaire HAL, est destinée au dépôt et à la diffusion de documents scientifiques de niveau recherche, publiés ou non, émanant des établissements d'enseignement et de recherche français ou étrangers, des laboratoires publics ou privés. 


\title{
ÉTUDES DE SAUTS DE FLUX LIMITÉS DANS LES SUPRACONDUCTEURS DE SECONDE ESPẼCE
}

\author{
L. BOYER, G. FOURNET, A. MAILFERT et J. L. NOEL (*)
}

I. Introduction. - Quand on applique parallèlement à la surface d'un cylindre supraconducteur de seconde espèce impur un champ magnétique croissant lentement $H_{0}(t)$, les lignes de tourbillon se créent à la surface et pénètrent dans le cylindre sur une distance qui dépend de $H_{0}$ et de la densité de courant critique $j(B, T)$. La connaissance de la densité critique (fonction de l'induction $B$ et de la température $T$ ) permet de déterminer la variation $B(r)$, où $r$ est la coordonnée radiale. Nous utiliserons, par la suite, la variable $u=R_{0}-r$ (Fig. 1), qui représente la distance mesurée à partir de la surface $\left(R_{0}\right.$ : diamètre du cylindre). Il est maintenant bien connu que sous certaines conditions, le profil $B(u)$ peut devenir instable. Un saut de flux se produit lorsque, sous l'effet d'une excitation extérieure infinitésimale, une pénétration finie de flux magnétique a lieu dans le cylindre. Le saut de flux est limité lorsqu'à la fin du phénomène un nouveau profil d'équilibre $B(u)$ est atteint, et il est complet si l'induction est alors égale en tout point à l'induction à la surface.

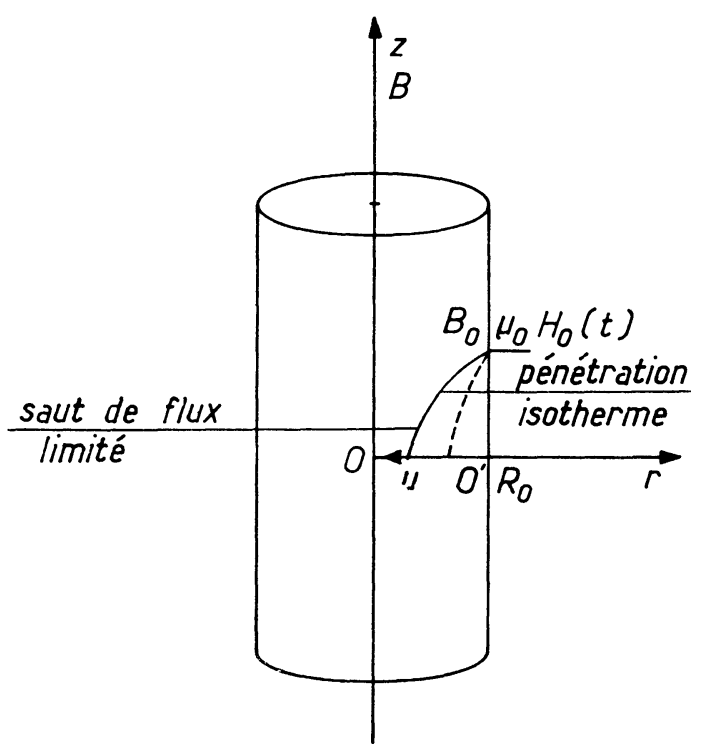

FIG. 1. - Géométrie utilisée dans l'étude des sauts de flux et de la pénétration isotherme du flux.

(*) L. C. I. E.-L. G. E., B. P. 8，92-Fontenay-aux-Roses, France.
Les sauts de flux ont été étudiés théoriquement pour des échantillons de faible dimension [1], [2] ou des échantillons semi-infinis [3], [4]. Nous avons cherché à nous rapprocher de ce dernier cas en étudiant des échantillons cylindriques de dimensions suffisantes. Dans cette géométrie il n'existe que peu de résultats expérimentaux concernant les répartitions d'induction et la dynamique des sauts de flux.

Le travail que nous présentons comprend deux parties : dans la première, nous rappelons les résultats d'expériences donnant la répartition de l'induction dans l'échantillon après des sauts de flux limités; dans la seconde, nous proposons un modèle théorique pour la propagation de l'instabilité, modèle qui nous permet d'obtenir l'évolution de la température et de l'induction à tout moment au cours du saut de flux.

II. Répartition de l'induction dans un échantillon après un saut de flux limite. - Nous déclenchons les sauts de flux dans notre échantillon en superposant à un champ constant et uniforme auquel correspond une valeur d'induction de surface $B$ une impulsion ou un échelon de champ magnétique. Après un temps suffisamment long pour que la température dans le barreau soit de nouveau la température initiale, nous appliquons, pour connaître la répartition de l'induction, la technique du «balayage isotherme » [5] dont nous rappelons le principe : si dans un cylindre supraconducteur il existe un flux piégé caractérisé par une répartition de l'induction $B_{\mathrm{p}}$ suivant l'axe ne dépendant que de $u$ et si on applique à ce cylindre, parallèlement aux génératrices, un champ magnétique croissant linéairement dans le temps, assez lentement pour que le système reste isotherme, on observe à la surface un champ électrique dont le module peut être relié [6] à $B_{\mathrm{p}}(u)$ lorsque l'on connaît la densité de courant critique $j(B, T)$ du matériau.

L'avantage principal de cette méthode est de donner la courbe $B_{\mathrm{p}}(u)$ avec une excellente précision spatiale sans avoir à pratiquer de fente dans l'échantillon.

Les figures 1 à 3 représentent les répartitions $B_{\mathrm{p}}(u)$ obtenues pour un échantillon cylindrique de $\mathrm{NbTi}$. Les caractéristiques principales des sauts de flux limités observés sont les suivantes :

- l'écart entre la courbe de pénétration isotherme avant saut de flux et la courbe relevée après saut de 
flux croît lorsque la valeur $B_{0}$ du champ extérieur au moment du saut de flux croît (Fig. 1) ;

- pour une valeur donnée de $B_{0}$ et pour des sauts de flux d'amplitude suffisante, le profil observé après saut de flux est pratiquement indépendant de la température initiale $T_{0}$ du déclenchement (Fig. 2);

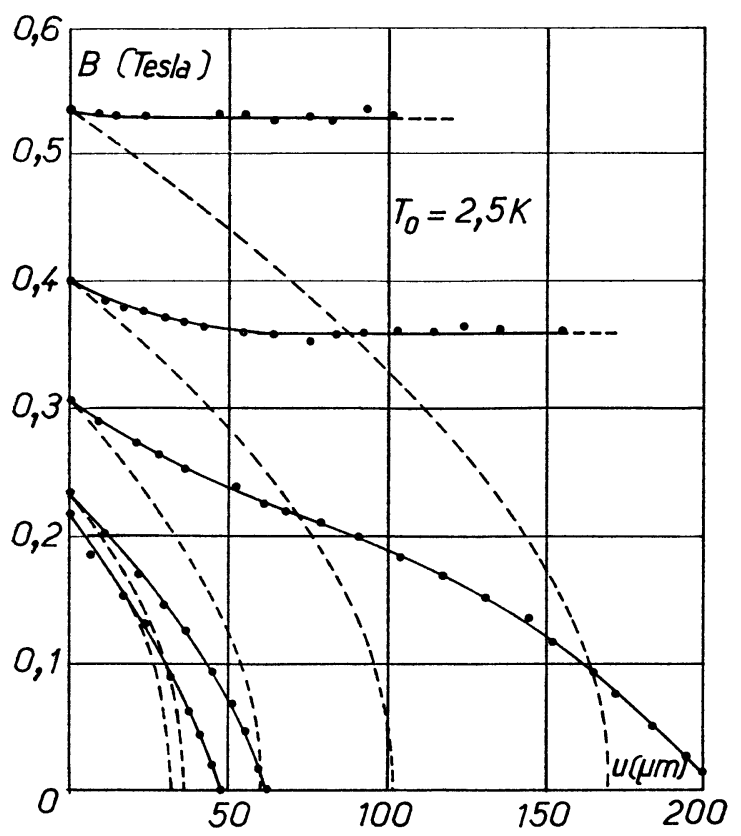

Fig. 2. - Courbes expérimentales $B(u)$ obtenues à $2,5^{\circ} \mathrm{K}$ dans l'hélium liquide. Trait discontinu : pénétration isotherme. Trait continu : sauts de flux limités.

- pour un couple de valeurs $\left(B_{0}, T_{0}\right)$ donné, l'amplitude du saut de flux dépend de la nature de l'échange thermique avec le milieu extérieur (supraconducteur en contact avec de l'hélium liquide ou gazeux, Fig. 3).

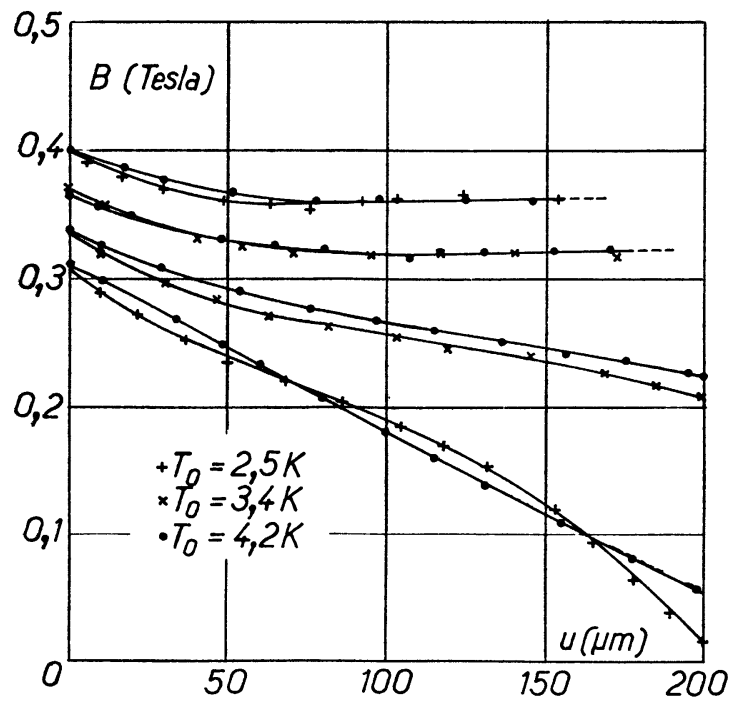

FIG. 3. - Influence de la température initiale sur la courbe $B(u)$ après saut de flux.

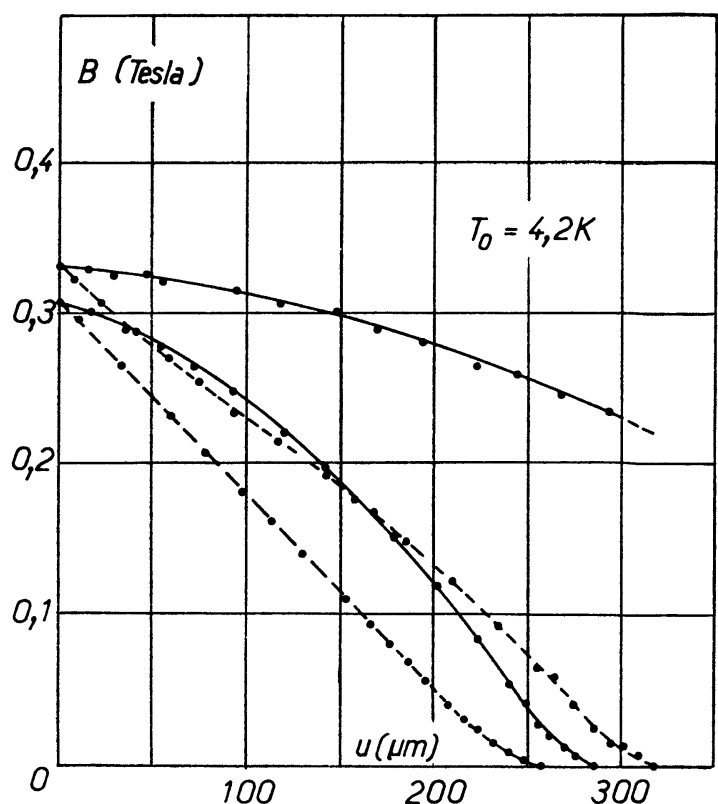

Fig. 4. - Influence de l'environnement. Trait discontinu : hélium liquide à $4,2^{\circ} \mathrm{K}$. Trait continu : hélium gazeux à $4,2^{\circ} \mathrm{K}$.

Notre méthode fournit également des renseignements sur la répartition spatiale $T_{\mathrm{f}}(u)$ des températures dans l'échantillon à la fin du saut de flux. En effet, à ce moment, les lignes de tourbillon cessent de se déplacer (en un point où l'induction est $B$ ) pour une température $T_{\mathrm{f}}$ telle que la densité de courant soit égale à la valeur critique $j\left(B, T_{\mathrm{f}}\right)$. L'analyse des courbes $B_{\mathrm{p}}(u)$

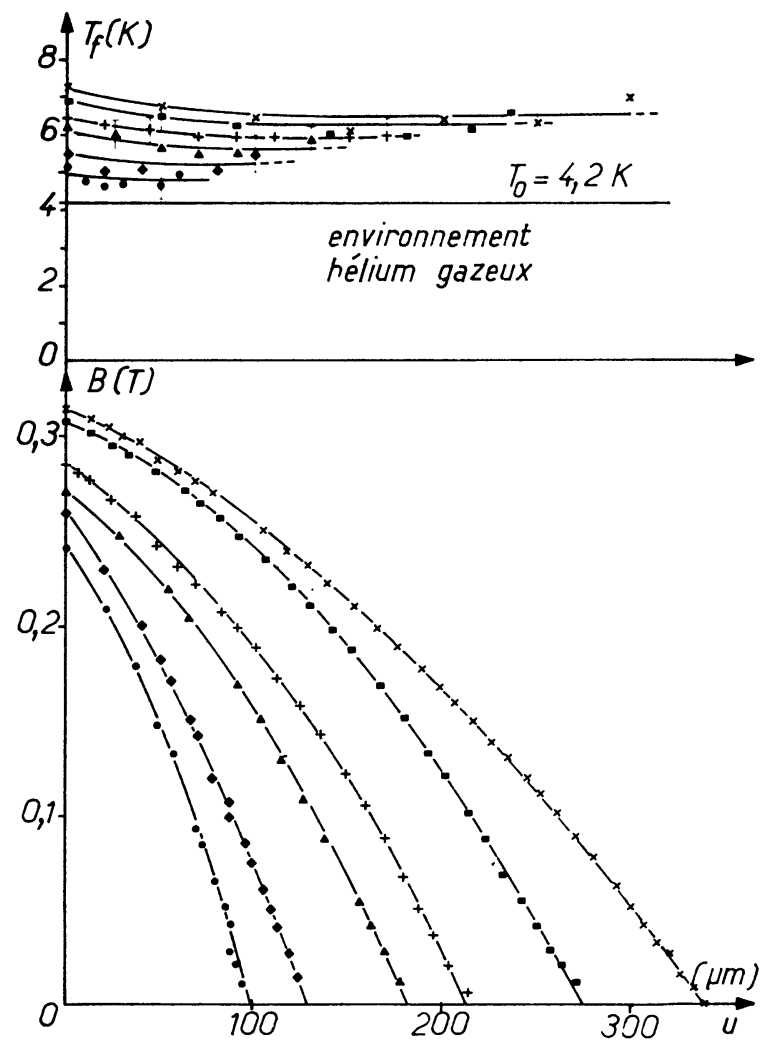

Fig. 5. - Variations de la température et de l'induction pour une série de sauts de flux à $4,2^{\circ} \mathrm{K}$ (hélium gazeux). 
permet donc de connaître la répartition $T_{\mathrm{f}}(u)$ au moyen de

$$
\frac{\mathrm{d} B_{\mathrm{p}}(u)}{\mathrm{d} u}=-\mu_{0} j\left[B_{\mathrm{p}}(u), T_{\mathrm{f}}(u)\right] .
$$

Ce travail, effectué par exemple sur une série de sauts de flux limités, pour une température de $4,2^{\circ} \mathrm{K}$ (milieu ambiant : hélium gazeux) conduit à la série de courbes de répartition représentée sur la figure 5 . On vérifie que la température finale atteinte est plus élevée pour les sauts de flux de grande amplitude. On observe également que la température $T_{\mathrm{f}}$ (aux erreurs de détermination près) est faiblement décroissante, pratiquement constante ou même présente un minimum, sur la distance correspondant à la pénétration du flux magnétique.

Ce résultat n'est pas en accord avec les prédictions théoriques de Swartz et Bean [4] décrivant les sauts de flux à partir de l'état critique adiabatique. Dans ce modèle, la température finale atteinte à l'extrémité interne du saut de flux $\left(B_{\mathrm{p}}=0\right)$ doit être égale à la température initiale. Notre résultat indique que la perturbation thermique s'est propagée plus profondément dans le barreau que la perturbation magnétique.

III. Propagation de l'instabilité. - De préférence à la description fondée sur l'hypothèse des états critiques adiabatiques [4], nous proposons pour expliquer la dynamique des sauts de flux le modèle suivant, partiellement en accord avec nos résultats précédents :

1. A tout moment durant l'instabilité, l'élévation de température est supposée se propager beaucoup plus loin que la variation d'induction : nous calculerons au moyen des lois générales de propagation de la chaleur l'évolution en fonction du temps de la température $T(u, t)$ dans le matériau en assimilant la dissipation d'énergie produite par le déplacement des lignes de tourbillon à un flux de chaleur $f(t)$ appliqué à la surface du matériau. Nous négligerons dans la suite les échanges thermiques avec l'extérieur.

Avec les approximations valables pour de nombreux supraconducteurs,

$$
\begin{aligned}
& C(T)=\alpha T^{3} \\
& K(T)=\beta T^{3}
\end{aligned}
$$

(où $\alpha$ et $\beta$ sont des constantes), un traitement voisin de celui où $C$ et $K$ sont constants [7] permet d'obtenir la valeur de la température au moment $t$ en un point à la distance $u$ de la surface :

$$
\begin{aligned}
& T(u, t)= \\
& =\left[T_{0}^{4}+4(\alpha \beta \pi)^{-1 / 2} \int_{0}^{t} f(t-\tau) \mathrm{e}^{-\alpha u^{2} / 4 \beta \tau} \frac{\mathrm{d} \tau}{\tau^{1 / 2}}\right]^{1 / 4}
\end{aligned}
$$

où $T_{0}$ est la température initiale, supposée uniforme dans le matériau.
La température à la surface s'écrit simplement :

$T(O, t)=\left[T_{0}^{4}+4(\alpha \beta \pi)^{-1 / 2} \int_{0}^{t} f(t-\tau) \frac{\mathrm{d} \tau}{\tau^{1 / 2}}\right]^{1 / 4}$.

2. A chaque instant la température est supposée uniforme et égale à $T(O, t)$ dans toute région où l'induction varie. L'utilisation de l'hypothèse des états critiques isothermes permet (en négligeant la variation de $B_{0}$ au cours du saut de flux) de relier la variation de température au flux de chaleur $f(t)$ produit par la pénétration de l'induction, ainsi qu'au champ électrique de surface $E(O, t)$. En supposant que le saut se développe essentiellement après la fin de l'impulsion ou de l'échelon, on obtient d'après [6]:

$$
\begin{aligned}
& E(O, t)=\frac{\mathrm{d} T(O, t)}{\mathrm{d} t} \times \\
& \times \int_{B_{0}}^{0} \int_{B_{0}}^{v} \frac{\partial}{\partial T}\left[\frac{1}{\mu_{0} j(w, T)}\right] \mathrm{d} w \mathrm{~d} v
\end{aligned}
$$

(où $v$ et $w$ ont la dimension d'une induction).

Cette expression se simplifie lorsque la densité critique a la forme particulière d'un produit de fonctions :

$$
j(B, T)=g(B) \cdot h(T) .
$$

On obtient alors :

$$
E(O, t)=-\frac{1}{h^{2}(T)} \frac{\mathrm{d} h}{\mathrm{~d} T} \frac{\mathrm{d} T}{\mathrm{~d} t} S_{\mathrm{i}}\left(B_{0}\right)
$$

où $s_{\mathrm{i}}\left(B_{0}\right)$ ne dépend que de l'induction extérieure et est aisément calculable pour un matériau obéissant à (6).

De même le flux de chaleur produit est évalué en retranchant au flux de puissance électromagnétique entrant : $\mu_{0}^{-1} E(O, t) B_{0}$ la dérivée par rapport au temps de l'énergie magnétique stockée dans le matériau

$$
\left(2 \mu_{0}\right)^{-1} \int_{u} B^{2}(u, t) \mathrm{d} u
$$

Pour un matériau obéissant à (6) le flux de chaleur s'écrit

$$
f(t)=s_{2}\left(B_{0}\right) E(O, t)
$$

où $s_{2}\left(B_{0}\right)$ est une fonction ne dépendant que de $B_{0}$.

Dans notre modèle, les solutions non triviales $\mathrm{du}$ système constitué par les équations (4), (7) et (8) donnent les variations, en fonction du temps, de la température " de surface " et du champ électrique. A partir de la mesure du champ électrique $(E O, t)$, au cours du saut de flux, on peut donc déterminer la variation de température $T(O, t)$ par deux voies différentes :

1) en intégrant (7),

2) en intégrant (4) à l'aide de (8) ;

la validité de notre modèle dépendra de l'accord des deux résultats. 


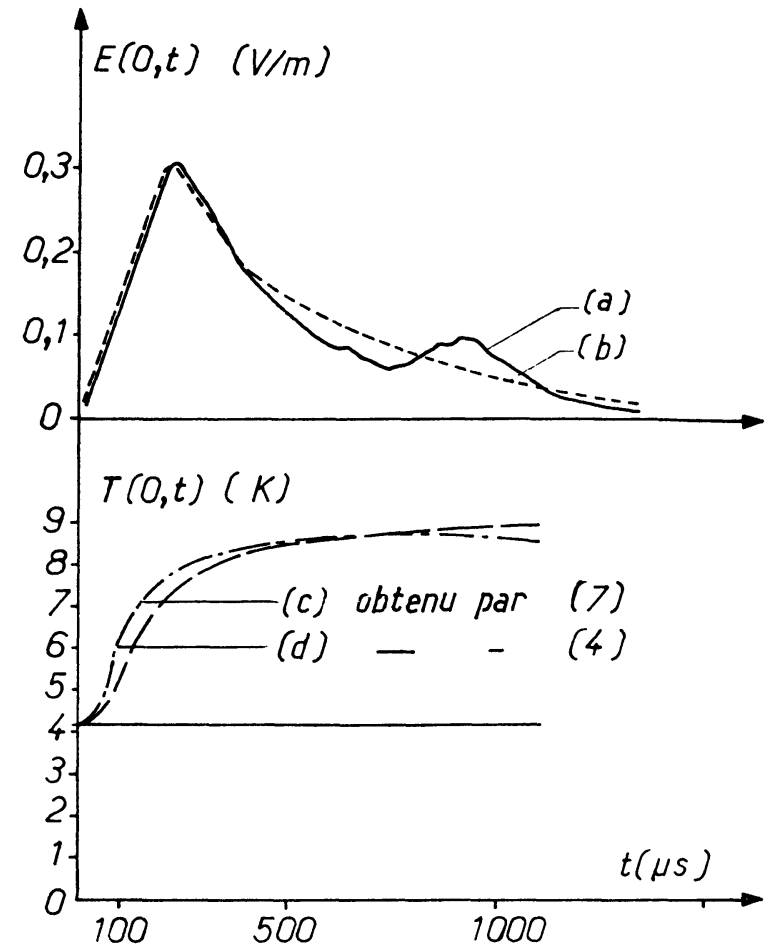

Fig. 6. - Dynamique d'un saut de flux. $T_{0}=4,2^{\circ} \mathrm{K}, B_{0}=0,37 \mathrm{~T}$ (Environnement : hélium gazeux).
Les résultats d'une expérience réalisée sur le même échantillon (cylindre de NB $40 \% \mathrm{Ti}$ ) sont représentés sur la figure 6. Le saut de flux a été déclenché par un échelon de champ de hauteur $16 \times 10^{3} \mathrm{~A} . \mathrm{m}^{-1}$ et de temps de montée égal à $100 \mu$ s.

On a représenté sur la figure : $a$ ) la courbe expérimentale $E(O, t)$ dont la forme irrégulière est probablement due aux inhomogénéités de champ et aux irrégularités géométriques de l'échantillon; $b$ ) la courbe lissée que nous avons utilisée dans le calcul ; $c$ ) et $d$ ) les résultats obtenus pour $T(O, t)$.

Nous n'avons pas trouvé, dans la littérature, de valeurs concernant la conductivité thermique et la chaleur spécifique du $\mathrm{Nb} 40 \% \mathrm{Ti}$; en utilisant pour $C(T)$ la valeur donnée dans [8] pour le $\mathrm{Nb} 44 \% \mathrm{Ti}$ et pour $K(T=4,2)$ la valeur donnée dans [9] pour le $\mathrm{Nb} 67 \%$ Ti l'accord des deux courbes de température semble assez bon. L'écart observé peut avoir plusieurs origines: géométrie cylindrique et non semi-infinie ; effets de bouts, ou plus probablement les approximations faites (2) sur les caractéristiques thermiques de notre échantillon.

Remerciements. - Ce travail a été effectué grâce au soutien apporté par la D. G. R. S. T. et les membres d'un club comprenant l'air liquide, Alsthom, C. E. M., C. G. E., E. D. F., Jeumont-Schneider.

\section{Bibliographie}

[1] Hancox (R.), Phys. Letters, 1965, 16, 208.

[2] Yamafuji (K.), Takeo (M.), Chikaha (J.), Yano (N.), IrIE (F.), J. Phys. Soc. Japan, 1969, 26, 315.

[3] WiPf (S. L.), Phys. Rev., 1967, 161, 404.

[4] Swartz (P. S.), Bean (C. P.), J. Appl. Phys., 1968, 39, 4991.

[5] Noel (J. L.), MAILfert (A.), Fournet (G.), J. Physique, 1971, 32, 171.
[6] Mailfert (A.), Thèse, Paris 1969, et Rev. Gén. Elec., 1969, 78, 12.

[7] Carslaw (H. S.) and Jaeger (J. C.), Conduction of heat in solids, Oxford Univ. Press, 1959, 76.

[8] Hampshire (R.), Sutton (J.), Taylor (M. T.), Proc. on Conf. on low temperatures, Londres, mars 1969.

[9] Dubeck (L.), Setty (K. S. L.), Phys. Letters, 1968, 27, 6. 